\title{
Identificación de una deleción causante de tumor del estroma gastrointestinal (Gist) por el análisis de los genes KIT y PDGFRA
}

\author{
José Buleje-Sono ${ }^{1, a}$, Oscar Acosta ${ }^{1,4, b}$, María Luisa Guevara-Fujita1,c, Luis Taxa ${ }^{2, d}$, Ricardo Fuj ita ${ }^{1, e}$
}

\section{RESUMEN}

Objetivo: Optimizar la detección de mutaciones en los genes KIT y PDGFRA en una muestra de tumor del estroma gastrointestinal (GIST).

Material y Métodos: Se analizó una muestra de tumor GIST fijada y embebida en parafina. Mediante la técnica de reacción en cadena de la polimerasa (PCR) se amplificaron los exones 9, 11, 13 y 17 del gen KIT y los exones 12 y 18 del gen PDGFR que contienen las mutaciones causales de GIST. Se confirmó la amplificación mediante electroforesis en gel de agarosa al $2 \%$. Los fragmentos amplificados, fueron purificados y secuenciados en un analizador genético. Se detectó una sobreposición de bases propio de una deleción por lo que se tuvo que clonar los productos del exón 11 para identificar el alelo mutado. Resultados: Se determinó la presencia de una mutación patogénica en el exón 11 del gen KIT. Dicha mutación es una deleción de 15 pares de bases que genera la pérdida de 5 aminoácidos en el receptor tirosina kinasa KIT. Se encontraron polimorfismos neutrales en los exones 11 del gen KIT y en el exón 18 del gen PDGFRA.

Conclusión: El análisis molecular mediante secuenciación automática, permitió identificar una mutación en el gen KIT en una muestra de tumor GIST. Esta técnica puede ser aplicada para caracterizar las mutaciones genéticas de casos peruanos de GIST y así poder establecer un tratamiento adecuado según su perfil mutacional. (Horiz Med 2014; 14(4): 43-47)

Palabras clave: Proteína Proto-Oncogénica c-KIT, Receptor alfa de Factor de Crecimiento Derivado de Plaquetas, Tumores del Estroma Gastrointestinal. (Fuente: DeCS BIREME).

identification of a causative deletion of gastrointestinal stromal tumor (gist) by the analysis of the KIT and PDGFRA genes

\begin{abstract}
Objective: To optimize the detection of mutations in the genes KIT and PDGFRA in a sample of gastrointestinal stromal tumor (GIST).

Material and Methods: We analyzed a GIST tumor sample fixed and embedded in paraffin. Using the technique of the polymerase chain reaction (PCR) we amplified exons 9, 11, 13 and 17 of the KIT gene and exons 12 and 18 PDGFR gene which contain tha causal mutations of GIST. PCR amplification was confirmed by gel electrophoresis on $2 \%$ agarose. The amplified fragments were purified and cloned for subsequent sequencing. An overlap of baeses, characteristic of a deletion was detected, so we had to clone the exon 11 products to identify mutated allele.

Results: We determined the presence of a pathogenic mutation in exon 11 of KIT gene. The mutation is a deletion of 15 base pairs and generates a loss of 5 amino acids in the KIT receptor tyrosine kinase. Neutral polymorphisms were also found in exon 11 of KIT gene and exon 18 of PDGFRA gene.

Conclusion: Molecular analysis by automatic sequencing identified a mutation in the KIT gene in a tumor sample GIST. This technique can be applied to characterize genetic mutations Peruvian cases of GIST and thus establish adequate treatment by mutational profile. (Horiz Med 2014; 14(4): 43-47)
\end{abstract}

Key words: Proto-Oncogene Protein c-KIT, Alpha Receptor Platelet-Derived Growth Factor, Gastrointestinal Stromal Tumors. (Source: MeSH NLM).

\footnotetext{
Centro de Genética y Biología Molecular. Facultad de Medicina Humana. Universidad de San Martín de Porres. Lima, Perú Laboratorio de Patología. Facultad de Medicina Humana. Universidad de San Martín de Porres. Lima, Perú Instituto Nacional de Enfermedades Neoplásicas. Lima, Perú

Laboratorio de Biología Molecular. Facultad de Farmacia y Bioquímica. Universidad Nacional Mayor de San Marcos. Lima, Perú
} 


\section{INTRODUCCIÓN}

Los tumores del estroma gastrointestinal (GIST, Gastrointestinal Stromal Tumors), son las neoplasias mesenquimales más comunes del tracto gastrointestinal (1), ubicándose principalmente dentro de la pared muscular del estómago y del intestino delgado y otros sitios anatómicos en la cavidad abdominal (2). Aproximadamente el 95\% de los GISTs expresan el receptor Tirosina quinasa KIT (CD117), el cual es utilizado en la discriminación de estos tumores de otros sarcomas que se desarrollan en el abdomen $(3,4)$.

Se piensa que los tumores GIST se originan de las células intersticiales de Cajal (ICCs).

Las ICCs están localizadas dentro y alrededor del plexo mientérico y funcionarían como células marcapasos intestinales que regulan la motilidad intestinal. Históricamente, los GISTs eran mal clasificados como leiomiomas o leiomiosarcomas.

Posteriormente, se ha determinado que los GISTs tienen diferentes características ultraestructurales y marcadores inmunofenotípicos, comparados con células normales y tumores de músculo liso (5).

Los GISTs, a menudo, portan mutaciones que activan el receptor tirosina quinasa KIT o el receptor del factor de crecimiento derivado de plaquetas (PDGFRA). En el caso del gen KIT, los cuatro exones involucrados: 9, 11, 13 y 17, corresponden a los dominios extracelular, juxtamembranoso, tirosina quinasa 1 y tirosina quinasa 2 de la proteína KIT, respectivamente.

Mutaciones en estos exones generan una activación constitutiva de la actividad tirosina quinasa de la proteína KIT, el principal evento oncogénico en estos tumores (6-9).

En aquellos casos que no presentan mutaciones en el gen KIT, se ha encontrado que algunos presentan mutaciones en el gen PDGFRA, el cual también es un miembro de la familia Tirosina quinasa tipo III. Mutaciones en este gen, en GISTs, resultan en la activación constitutiva de esta actividad tirosina quinasa, la cual contribuye a la formación del tumor (10).
La mayoría de mutaciones en el gen PDGFRA relacionadas con tumores GISTs, han sido reportadas en los exones 12 y 18 . Hay que tener en cuenta que las mutaciones en los exones de estos genes, son mutuamente excluyentes en la mayoría de tumores GISTs (10).

Aproximadamente, el $75 \%$ de las mutaciones en el gen KIT involucran el exón 11, agrupándose en los extremos 5' y 3' de dicho exón, que codifica el dominio Juxtamembranoso (citosólico) de la proteína KIT.

Las mutaciones, en el extremo 5' frecuentemente, incluyen deleciones internas y sustituciones de un solo aminoácido, mientras que en el extremo 3', se observa una mayor proporción de duplicaciones. Clínicamente, estos pacientes tienen tumores GIST gástricos con un curso indolente (11).

En comparación, un curso clínico agresivo con un elevado riesgo de recurrencia y corta sobrevida, se ha observado en pacientes con tumores GIST portadores de deleciones en el exón 11, mientras que en pacientes GIST con otro tipo de mutaciones e inclusive sin mutación, se ha observado una muy baja sobrevida libre de enfermedad $(12,13)$.

El objetivo del presente trabajo, fue optimizar la detección de mutaciones en los genes KIT y PDGFRA en una muestra de GIST.

\section{MATERIAL Y MÉTODOS}

Se evaluó una muestra de GIST, fijado en formalina al $10 \%$ e incluido en bloque de parafina. Se realizaron cortes histológicos de $5 \mu \mathrm{m}$ y tinción con hematoxilina-eosina.

El análisis inmunohistoquímico indicó que la muestra era positiva para la expresión de CD117.

Extracción y amplificación del DNA genómico El DNA genómico, fue extraído usando el kit High Pure PCR Template Preparation kit (ROCHE) siguiendo las indicaciones del fabricante. La cuantificación del DNA se realizó mediante el uso del fluorómetro QubitTM (InvitrogenTM). 
El DNA extraído, fue amplificado mediante la reacción en cadena de la polimerasa (PCR) usando primers diseñados para los exones: $9,11,13$ y 17 del gen KIT y los exones 12 y 18 del gen PDGFRA (Tabla 1). Las condiciones de PCR fueron: $95^{\circ} \mathrm{C}$ por 5 minutos, seguido de 35 ciclos a $95^{\circ} \mathrm{C}$ por 45 segundos, $55^{\circ} \mathrm{C}$ a $57^{\circ} \mathrm{C}$ durante 45 segundos (temperaturas específicas de hibridación en la tabla 1) y $72^{\circ} \mathrm{C}$ por 1 minuto.

Finalmente, una etapa de extensión a $72^{\circ} \mathrm{C}$ durante 10 minutos. La comprobación del proceso de amplificación se realizó mediante una electroforesis horizontal en gel de agarosa al $2 \%$. Los tamaños relativos de cada exón de ambos genes se encuentran detallados en la Tabla 1.

Tabla 1.- Lista de primers usados y condiciones para amplificación de los genes KIT y PDGFRA.

\begin{tabular}{|c|c|c|c|c|}
\hline Gen & Exón & Primer $\left(5^{\prime} \rightarrow 3^{\prime}\right)$ & $\begin{array}{l}\text { Ampl } \\
\text { (bp) }\end{array}$ & $\mathrm{Tm}$ \\
\hline \multirow{4}{*}{ KIT } & 9 & $\begin{array}{l}\text { F: 5'-tcctagagtaagccagggett-3', } \\
\text { R: } 5 \text { '-tggtagacagagcctaaacatc-3' }\end{array}$ & 284 & $55^{\circ} \mathrm{C}$ \\
\hline & 11 & $\begin{array}{l}\text { F: 5'-ctctctccagagtgetctaatgac-3' } \\
\text { R: } 5 \text { '-gttcettaaagtcactgttatgtgtacc-3' }\end{array}$ & 267 & $57^{\circ} \mathrm{C}$ \\
\hline & 13 & $\begin{array}{l}F: 5^{\prime} \text {-gacatcagttgccagttgt-3', } \\
R: 5^{\prime} \text {-Tgttttgataacetgacagac-3, }\end{array}$ & 214 & $57^{\circ} \mathrm{C}$ \\
\hline & 17 & $\begin{array}{l}\text { F: 5'-atggttttctttctcetcc-3' } \\
\text { R: 5'-tacattatgaaaatcacagg-3' }\end{array}$ & 243 & $57^{\circ} \mathrm{C}$ \\
\hline \multirow{2}{*}{ PDGFRA } & 12 & $\begin{array}{l}\text { F: 5'-ctggtgcactgggacttggtaat-3', } \\
\text { R: } 5 \text { '-gtgtgcaagggaaaagggagtct-3, }\end{array}$ & 235 & $55^{\circ} \mathrm{C}$ \\
\hline & 18 & $\begin{array}{l}\text { F: 5'-gggtgatgctattcagctacagatgg-3', } \\
\text { R: 5'-ccgaatctctagaagcaacacctgac-3' }\end{array}$ & 267 & $55^{\circ} \mathrm{C}$ \\
\hline
\end{tabular}

Secuenciación del DNA

Los 6 fragmentos amplificados por PCR, fueron purificados con el kit QIAquick PCR Purification (QIAGEN). Posteriormente, amplificados con el kit BigDye Terminator versión 3.1 Cycle sequencing y secuenciados en ambas direcciones (forward $y$ reverse) en un analizador genético $A B I 3500$ (Applied Biosystems).

Se realizaron dos procesos de secuenciación. La primera sirvió para determinar la presencia de mutaciones en los genes KIT y PDGFRA. Una posible mutación se encontró en el exón 11 del gen KIT con una sobreposición de bases típico de una deleción/ inserción en uno de los alelos.

Análisis bioinformático

El análisis de las secuencias, se realizó mediante el uso del software SerialCloner (V1.3r11) (http:// www.serialbasics.com). Las mutaciones se describieron en base a las secuencias de referencia NM_000222 y NM_006206 para los genes KIT y PDGFRA, respectivamente.

Clonación de productos de PCR

Para diferenciar entre la secuencia normal y mutada (posible deleción/ duplicación), se clonó el fragmento amplificado correspondiente al exón 11 del gen KIT utilizando el set de reactivos $\mathrm{PCR} \otimes 8$ / GW/TOPO $\otimes T A$ Cloning (Invitrogen) y siguiendo las indicaciones del fabricante. Brevemente, los productos de PCR se ligaron en el vector plasmídico $\mathrm{PCR} \otimes 8 / \mathrm{GW} / \mathrm{TOPO} \otimes$, las células Electro Max TM DH10BTM-T1® fueron transformadas con los constructos generados, estas células se cultivaron en medio LB con el antibiótico espectinomicina e incubadas a $37 \mathrm{C}$.

El ADN del plásmido, se extrajo usando el método modificado de lisis alcalina/PEG y luego se sometió a digestión con la enzima EcoR1, electroforesis en agarosa $1 \%$ y tinción con bromuro de etidio para confirmar la presencia del fragmento clonado.

Los plásmidos obtenidos fueron secuenciados en ambos sentidos para confirmar las variaciones de la secuencia.

\section{RESULTADOS}

Se analizó una muestra de GIST fijada y embebida en parafina. En el análisis inmuno histoquímico, se determinó la sobreexpresión del marcador CD117 (datos no mostrados). La muestra provenía de una paciente de 67 años con tumoración a nivel de estómago.

El análisis molecular de los exones: 9, 11, 13 y 17 del gen KIT y los exones 12 y 18 del gen PDGFRA, permitió la detección de una mutación heterocigota (deleción/inserción) en el exón 11 del gen KIT. 
Después de la clonación con el kit pCR®8/GW/ TOPO $₫ T A$ Cloning $₫$ para individualizar y secuenciar separadamente los alelos se encontró la mutación, denominada p.K550_E554del, que corresponde a una deleción de 15 pares de bases a nivel del extremo 5' del exón 11, generando una pérdida de cinco aminoácidos (Lys-Pro-Met-Tyr-Glu) entre los codones 550 y 554 . Esta mutación afecta la secuencia aminoacídica del dominio yuxtamembranoso del receptor tirosina kinasa. También se encontró un cambio puntual (c.1752T>C) en el codón 584 del exón 11, que no genera un cambio de aminoácido, por lo que se considera un polimorfismo neutral.

En el caso de los exones 12 y 18 del gen PDGFRA no se encontraron mutaciones patogénicas, sólo en el codón 824 del exón 18 se encontró un polimorfismo neutral (c.2472C>T) que no genera un cambio de aminoácido.

\section{DISCUSIÓN}

Las mutaciones en los genes KIT y PDGFRA, en tumores del estroma gastrointestinal, pueden causar una proliferación celular descontrolada y una inhibición de la apoptosis mediante producción de señales continuas. En orden de frecuencia decreciente, estas mutaciones están presentes en los exones: $11,9,13$ y 17 del gen KIT, y en los exones 18 y 12 del gen PDGFRA $(14,15)$.

En el gen KIT, estas mutaciones resultan en la activación del dominio kinasa de la proteína KIT, independientemente del ligando. La mutación p.K550_E554del encontrada en nuestro estudio, ya ha sido reportada y estaría afectando la estructura del dominio juxtamembranoso de la proteína que tiene como función prevenir la activación constitutiva de la kinasa $(16,17)$.

Esta mutación, es una deleción de 15 pares de bases que estaría asociada con una corta sobrevida libre de progresión (18).

Las deleciones que implican el codón 557 y/o codón 558 están asociadas con un comportamiento maligno (19). Se han reportado casos en las que la deleción se ubica entre los codones 550 y 558 (p.K550_K558del), con una frecuencia variable (1.4 a 1.6\%) y estaban asociadas con un comportamiento agresivo $(10,20)$, por lo que podemos esperar que en nuestro caso, las características del tumor deberían ser similares, ya que la mutación se encuentra en la misma región.

El estado mutacional del gen KIT, es también un factor predictivo de respuesta a tratamiento con un inhibidor selectivo de tirosina kinasa (20-21).

La ausencia de mutaciones en el gen PDGFRA en la muestra analizada, se corresponde con el hecho de que las vías oncogénicas de KIT y PDGFRA son alternativas y mutuamente excluyentes en el desarrollo de GISTs (14).

Finalmente, en la versión 2012 de las Guías de Práctica Clínica de la ESMO (European Society for Medical Oncology) para GIST, se indica que la caracterización molecular de la mutación debe ser obligatoria para todos los pacientes con GIST, con el fin de orientar en la administración de un tratamiento adyuvante $(22,23)$.

En conclusión, el análisis molecular mediante secuenciación automática, permitió identificar una mutación en el gen KIT en una muestra de tumor GIST. Esta técnica puede ser aplicada para caracterizar las mutaciones genéticas de casos peruanos de tumores GIST para establecer un tratamiento y pronóstico adecuados según el perfil mutacional.

\section{Fuentes de financiamiento}

El estudio ha sido financiado por el laboratorio de Genética y Biología Molecular de la Facultad de Medicina Humana de la Universidad de San Martín de Porres.

\section{Conflictos de interés}

Los autores declaran no tener ningún conflicto de interés. 


\section{REFERENCIAS BIBLIOGRÁFICAS}

1. Agaimy A. Gastrointestinal stromal tumors (GIST) from risk stratiffication systems to the new TNM proposal: more questions than answers? A review emphasizing the need for a standardized GIST reporting. Int J Clin Exp Pathol 2010; 3:461-471.

2. Joensuu H, Kindblom LG. Gastrointestinal stromal tumors: a review. Acta Orthop Scand Suppl 2004; 75:62-71.

3. Miettinen M, Lasota J, Sobin LH. Gastrointestinal stromal tumors of the stomach in children and young adults: a clinicopathologic, immunohistochemical, and molecular genetic study of 44 cases with long-term follow-up and review of the literature. Am J Surg Pathol 2005; 29:1373-1381.

4. Tzen C, Mau B. Analysis of CD117 negative gastrointestinal stromal tumors. World J Gastroenterol 2005; 11:1052-1055.

5. Gold J, Dematteo R. Combined Surgical and Molecular Therapy: The Gastrointestinal Stromal Tumor Model. Ann Surg 2006; 244: $176-184$.

6. Corless CL, Fletcher JA, Heinrich MC. Biology of gastrointestinal stromal tumors. J Clin Oncol 2004; 22:3813-25.

7. Tornillo L, Terracciano L. An update on molecular genetics of gastrointestinal stromal Tumours. J Clin Pathol 2006; 59:557-563.

8. Debiec-Rychter M, Dumez H, Judson I, et al. Use of c-KIT/PDGFRA mutational analysis to predict the clinical response to imatinib in patients with advanced gastrointestinal stromal tumors entered on phase I and II studies of the EORTC Soft Tissue and Bone Sarcoma Group. Eur J Cancer 2004; 40:689-95.

9. Rubin BP, Singer S, Tsao C, et al. KIT activation is a ubiquitous feature of gastrointestinal stromal tumors. Cancer Res 2001; $61: 8118-21$

10. Corless CL, Schroeder A, Griffith D, et al. PDGFRA mutations in gastrointestinal stromal tumors: frequency, spectrum and in vitro sensitivity to imatinib. J Clin Oncol 2005; 23:5357-64.

11. Kontogianni-Katsarou K, Dimitriadis E, Lariou C, Kairi-Vassilatou E, Pandis N, Kondi-Paphiti A. KIT exon 11 codon 557/558 de ᄀletion/ insertion mutations define a subset of gastrointestinal stromal tumors with malignant potential. World J Gastroenterol 2008; 14:1891-1897.

12. Michelucci A, Chiappetta C, Cacciotti J, Veccia N, Astri E, Leopizzi $M$, et al. The KIT Exon 11 Stop Codon Mutation in Gastrointestinal Stromal Tumors: What Is the Clinical Meaning? Gut Liver 2013; 7:35-40

\section{Correspondencia:}

José Luis Buleje Sono, PhD.

Dirección:Av. Alameda del Corregidor 1531, Urb. Los

Sirius, Las Viñas, La Molina, Lima 12.

Teléfono: 985225663

Correo electrónico: jbulejes@usmp.pe
13. Dematteo RP, Gold JS, Saran L, Gönen M, Liau KH, Maki RG, et al. Tumor mitotic rate, size, and location independently predict recurrence after resection of primary gastrointestinal stromal tumor (GIST). Cancer 2008; 112:608-615.

14. Miettinen M, Lasota J. Gastrointestinal Stromal Tumors Review on Morphology, Molecular Pathology, Prognosis, and Differential Diagnosis. Arch Pathol Lab Med 2006; 130:1466-78.

15. Heinrich MC, Corless CL, Duensing A, et al. PDGFRA activating mutations in gastrointestinal stromal tumors. Science 2003; 299:708-10.

16. Agaram NP, Besmer P, Wong GC, Guo T, Socci ND, Maki RG, et al. Pathologic and molecular heterogeneity in imatinib-stable or imatinib-responsive gastrointestinal stromal tumors. Clin Cancer Res 2007; 13(1):170-81.

17. Mol CD, Dougan DR, Schneider TR, Skene RJ, Kraus ML, Scheibe DN et al. Structural basis for the autoinhibition and STI 571 inhibition of c-Kit tyrosine kinase. J Biol Chem 2004; 279: 31655-31663.

18. Corless CL, Barnett CM, Heinrich MC. Gastrointestinal stromal tumors: origin and molecular oncology. Nat Rev Cancer 2011; 11(12):865-878.

19. Martin-Broto J, Gutierrez A, Garcia-del-Muro X, Lopez-Guerrero JA, Martinez-Trufero J, de Sande LM, et al. Prognostic time dependence of deletions affecting codons 557 and/or 558 of KIT gene for relapse-free survival (RFS) in localized GIST: a Spanish Group for Sarcoma Research (GEIS) Study. Ann Oncol 2010; 21(7):1552-1557.

20. Wozniak A, Rutkowski P, Piskorz A, Ciwoniuk M, Osuch C, Bylina E, et al. Prognostic value of KIT/PDGFRA mutations in gastrointestinal stromal tumours (GIST): Polish Clinical GIST Registry experience. Ann Oncol 2012; 23(2):353-60.

21. Muñoz C, Sabah S, Navarro A, Planzer M, Silva C, Santander R. Tumores del estroma gastrointestinal (GIST): Revisión de la literatura. Gastr Latinoam 2006; 17(1): 43-51.

22. Blay JY, Le Cesne A, Cassier PA, Ray-Coquard IL. Gastrointestinal stromal tumors (GIST): a rare entity, a tumor model for personalized therapy, and yet ten different molecular subtypes. Discov Med. 2012; 13(72):357-67.

23. ESMO/European Sarcoma Network Working Group. Gastrointestinal stromal tumors: ESMO Clinical Practice Guidelines for diagnosis, treatment and follow-up. Ann Oncol 2012; 23 (7): vii49 - vii55. 